\title{
Construção e Evidências de Validade do Questionário de Recursos e Demandas Laborais
}

\author{
Larissa Sanford Ayres Farinaํㅡ, Gabriel Dos Reis Rodrigues, Natália Kluwe Fagundes \\ Universidade Federal do Rio Grande do Sul, Porto Alegre-RS, Brasil \\ Thamires Casarotto Carafini \\ Universidade Luterana do Brasil, Canoas-RS, Brasil \\ Laisla Gabriele Conceição Soares Moreira \\ Centro Universitário FADERGS, Porto Alegre-RS, Brasil \\ Wagner De Lara Machado \\ Pontifícia Universidade Católica do Rio Grande do Sul, Porto Alegre-RS Brasil \\ Claudio Simon Hutz \\ Universidade Federal do Rio Grande do Sul, Porto Alegre-RS, Brasil
}

\section{RESUMO}

Apesar do modelo de Recursos e Demandas de Trabalho (RDT) gerar inúmeras pesquisas teóricas relevantes, não existe instrumento proposto que mensure os diversos recursos e demandas hipotetizados. Baseando-se no modelo RDT, esse estudo tem como objetivo a construção e validação do Questionário de Recursos e Demandas de Trabalho (QRDT). Os itens foram criados apoiados em uma análise de literatura e passaram por diversas reformulações. A versão final do instrumento continha 44 itens. O QRDT foi respondido por 317 participantes entre 20 e 65 anos. No R, uma análise fatorial exploratória (AFE) com rotação oblimin foi conduzida, extraindo-se oito fatores. O coeficiente alfa dos fatores foi igual ou acima de 0,80 , exceto para dois fatores. Não houve diferenças significativas entre homens e mulheres. Sugere-se que mais estudos utilizando o QRDT sejam produzidos, colaborando para uma validade mais robusta e contribuindo para a qualidade de vida no trabalho.

Palavras-chave: característica do trabalho; psicologia organizacional; psicometria.

\section{ABSTRACT - Development and Validity Evidences of the Job Demands and Resources Questionnaire}

Although the Job Demands-Resources (JD-R) model has generated many relevant theoretical studies, no instrument has been proposed to measures the various hypothesized demands and resources. Based on the JD-R model, this study aimed to construct and validate the Job Demands-Resources Questionnaire (JD-RQ). The items were developed based on a literature review and underwent several reformulations. The final version of the instrument contained 44 items. The JD-RQ was answered by 317 participants between 20 and 65 years of age. Exploratory Factor Analysis (EFA) with oblimin rotation was conducted, extracting 8 factors. The coefficient alpha values for the all the factors, except for 2 , were .80 or above. There were no significant differences between men and women. It is suggested that more studies using the JD-RQ are carried out, collaborating for more robust validity and contributing to the quality of life at work.

Keywords: job resources; organizational psychology; psychometrics.

RESUMEN - Construcción y Evidencias de Validez del Cuestionario de Demandas y Recursos Laborales

A pesar de que el modelo de Demandas y Recursos Laborales (DRL) ha generado innumerables investigaciones teóricas relevantes, todavía no existe un instrumento propuesto que valore los diversos recursos y demandas sugeridas. Basándose en el modelo DRL, este estudio tuvo como objetivo la construcción y validación del Cuestionario de Demandas y Recursos Laborales (CDRL). Los ítems fueron creados respaldados en un análisis de literatura y pasaron por diversas reformulaciones. La versión final del instrumento contiene 44 ítems. El CDRL fue respondido por 317 participantes entre 20 y 65 años. Con R, fue realizado un Análisis Factorial Exploratorio (AFE) con rotación oblicua, extrayendo 8 factores. El coeficiente alfa de los factores fue igual o superior a .80, excepto para dos factores. No hubo diferencias significativas entre hombres y mujeres. Se sugiere que sean realizados más estudios utilizando el CDRL, con el fin de obtener una validez más consistente, contribuyendo, de este modo, a la calidad de vida en el trabajo.

Palabras clave: característica del trabajo; psicología organizacional; psicometría.

Estudos no campo da Psicologia Positiva Organizacional e do Trabalho (PPOT) formulam que as relações positivas entre trabalhador e empresa contribuem tanto para a qualidade de vida do funcionário 
quanto para o sucesso da instituição (Erdogan, Bauer, Truxillo, \& Mansfield, 2012; Snyder \& Lopez, 2009; Taris \& Schaufeli, 2016). O Modelo de Recursos e Demandas de Trabalho (Modelo RDT) é uma proposta relevante para se mapear os possíveis obstáculos e catalisadores do vínculo colaborador/empresa (Bakker, Van Velhoven, \& Xanthopoulou, 2010; Bakker \& Demerouti, 2014; Farina, Rodrigues, \& Hutz, 2018; Mäkikangas, Bakker, Aunola, \& Demerouti, 2010; Schaufeli, Bakker, \& Van Rhenen, 2009; Schaufeli, 2017; Taris \& Schaufeli, 2016).

O Modelo RDT apresenta duas dimensões: os recursos e as demandas laborais. Os recursos são aspectos físicos, psicológicos, sociais ou organizacionais que amenizam as demandas. Os recursos têm a potência de gerar mais aprendizado, crescimento profissional e interesse pelo trabalho (Bakker \& Demerouti, 2014; Dal Vesco, Beuren, \& Popik, 2016; Mäkikangas et al., 2010; Schaufeliet al., 2009). Um exemplo de recurso poderia ser Comunicação, que compreende a eficiência das informações transmitidas que proporcionam o crescimento numa equipe (Cousins et al., 2004; Schaufeli et al., 2009)

Já as demandas são aspectos físicos, psicológicos, sociais ou organizacionais que exigem esforços do trabalhador (Bakker \& Demerouti, 2014; Schaufeli et al., 2009). No entanto, é interessante ressaltar que as demandas nem sempre são negativas, apenas quando requerem do indivíduo competências que ele não possui, tornando-se, nesse caso, um estressor (Bakker \& Demerouti, 2014). Um exemplo de demanda são conflitos interpessoais, que se refere às relações laborais tumultuosas. Tais relações podem comprometer a qualidade do trabalho ao gerarem estresse ao trabalhador, pois implicaria um custo psicológico do indivíduo sob essa demanda, podendo afetar seu desempenho (Hakanen \& Schaufeli, 2012; Schaufeli et al., 2009).

De acordo com estudos anteriores, os recursos de trabalho estão associados ao engajamento, ao estado de flow e à satisfação de vida (Erdoganet al., 2012; Taris \& Schaufeli, 2016). Assim sendo, o Modelo RDT pode servir como uma base relevante tanto para promoção de qualidade de vida do trabalhador quanto para a maximização de bons resultados da empresa e melhoria do clima organizacional (Bakker \& Demerouti, 2014; Schaufeli \& Bakker, 2004; Schaufeli, Dijikstra, \& Vazquez, 2013).

Ainda não há instrumento brasileiro de avaliação das características do trabalho que se baseie no modelo RDT, indicando que essa discussão teórica da PPOT ainda não adentrou ao campo empírico de avaliação no Brasil. Entretanto, existe uma gama de instrumentos no país que avaliam aspectos do trabalho, sem necessariamente apoiarem-se no modelo RDT. Para avaliar a liderança autêntica, por exemplo, há o Authentic Leadership Questionnaire (ALQ; Cervo, Mônico, Santos, Hutz, \& Pais, 2016). Tal questionário aborda aspectos pessoais importantes da liderança, estando os escores positivamente relacionados a índices de emoções positivas, inovação e efetividade do time. Logo, esse recurso individual parece impactar os recursos e as demandas laborais. Percebe-se, no entanto, que a ALQ tem um enfoque nos atributos pessoais, não fornecendo dados relevantes sobre o ambiente laboral e tendo sua aplicação limitada a líderes.

Além do ALQ, Jesus e Rowe (2014) adaptaram a Escala de Percepções de Justiça, que aborda a percepção de justiça organizacional. Tal construto está relacionado ao comprometimento e à satisfação no trabalho. Apesar dessa escala viabilizar a descrição de um trabalhador sobre a percepção de justiça organizacional, ela é limitada somente a esse aspecto do trabalho (i.e., justiça), informando dados parciais acerca dos recursos e demandas de trabalho que, de acordo com o modelo RDT, uma pessoa experimenta cotidianamente.

Considerando-se os recursos e demandas do trabalho como sendo relacionados, embora diferentes entre si, surge a necessidade de avaliá-los conjuntamente por meio de um instrumento validado para a cultura brasileira. Tal escala poderá oferecer valores sobre cada recurso e demanda que busque investigar, possibilitando um entendimento singularizado de tais fatores.

Ademais, esse instrumento possibilitaria uma compreensão ampla da percepção de um colaborador sobre seu ambiente de trabalho e proporcionaria informações úteis às organizações sobre possíveis mudanças que poderiam ser feitas na instituição. Por fim, tal instrumento deveria seguir o modelo RDT, teoria da PPOT que consegue integrar aspectos laborais positivos e negativos, garantindo um entendimento dinâmico do ambiente de trabalho. Além das questões supracitadas, essa escala que abrange vários recursos e demandas em um só questionário seria útil, pois facilitaria a integração desta a grandes pesquisas em combinação com uma bateria de outros testes ou a aplicação em contextos organizacionais (Nota \& Rossier, 2015).

Assim sendo, este estudo tem como objetivo geral aprimorar a avaliação dos recursos e demandas no contexto de trabalho. Isso posto, o objetivo específico deste estudo foi construir e validar um questionário baseado no Modelo de Recursos e Demandas de Trabalho de acordo com a teoria apresentada em pesquisas anteriores (Bakker \& Demerouti, 2014; Schaufeli \& Bakker, 2004; Schaufeli et al., 2013; Taris \& Schaufeli, 2016).

2 Embora haja autores que diferenciem os significados dos termos "organizações", "empresas" e "instituições", no presente estudo estes serão utilizados como sinônimos. 


\section{Método}

\section{Participantes}

A amostra total foi de 317 participantes, sendo que a maioria deles residia no Rio Grande do Sul $(n=228$; $71,9 \%)$ e era do sexo feminino $(n=212 ; 66,9 \%)$. A idade dos participantes variou entre 20 e 65 anos $(M=39,7$; $\mathrm{DP}=10,8)$. A escolaridade mínima dos participantes foi o ensino médio completo e a maior parte declarou possuir pós-graduação $(n=216 ; 68,1 \%)$.

\section{Construção e Validade de Conteúdo}

A construção e validade de conteúdo do questionário baseou-se nas diretrizes estabelecidas pela International Test Commission (2005). A Figura 1 sumariza o processo de formulação do QRDT. No início, houve amplo estudo da literatura a respeito de recursos e demandas (Bakker \& Demerouti, 2014; Farina et al. (2018); Mäkikangas et al., 2010; Schaufeli \& Bakker, 2004; Schaufeli et al., 2013; Taris \& Schaufeli, 2016). Após essa etapa, levantou-se os itens dos instrumentos Escala de Consciência Plena do Trabalho (Filho, Ferreira, \& Valentini, 2017), Questionnaire on the Experience and Evaluation of Work (Van Veldhoven \& Meijman, 1994), Job Crafting Questionnaire (Slemp \& Vella-Brodrick, 2013), versão brasileira da Utrecht Work Engagement Scale - UWES (Vazquez, Magnan, Pacico, Hutz, \& Schaufeli, 2015), Dutch Boredom Scale (Reijseger et al., 2013) e Maslach Burnout Inventory (Schaufeli, Bakker, Hoogduin, Schaap, \& Klader, 2001).

A partir do estudo desses itens e da literatura estudada do modelo RDT, o grupo de pesquisa desenvolveu uma lista de 15 fatores do ambiente laboral que foram recorrentemente observados. Tais fatores foram: conflitos de papel, conflitos interpessoais, suporte da liderança, suporte dos colegas, autonomia, clareza de papel, comunicação, congruência de valores, desafios apropriados, feedback imediato, justiça, mudança, perspectiva de crescimento, significado da tarefa, habilidades apropriadas no trabalho. Considerando-se esses fatores, e tendo como objetivo a compreensão dinâmica do ambiente laboral, 97 itens foram construídos ou adaptados das escalas citadas e a partir do estudo realizado por dois juízes independentes. Os fatores citados foram apenas utilizados na criação ou adaptação dos itens, e não permanecem na pesquisa após essa etapa.

Os itens foram analisados independentemente por quatro juízes, todos pós-graduados e/ou com experiência anterior de pelo menos dois anos em pesquisa. Após discussões, itens que não obtiveram consenso com relação a sua clareza de escrita foram reformulados. Itens semelhantes ou com significado muito próximo foram excluídos ou alterados. Após essa etapa, a nova lista passou a conter 45 itens. Esses itens foram, então, randomizados.

Um estudo piloto foi feito com uma amostra de 49 pessoas. O questionário foi disponibilizado na plataforma Survey Monkey para preenchimento, com alternativas de resposta do tipo Likert de cinco pontos entre "Discordo plenamente" e "Concordo plenamente". Dois juízes também responderam, colaborando com indicações e revisões importantes.

Com os resultados do estudo piloto, mais duas edições foram conduzidas no questionário. A escala tipo Likert passou de cinco para quatro pontos, o que impele o respondente a escolher entre "discordo" ou "concordo", eliminando a possibilidade da neutralidade. Ademais, a alternativa "Não se aplica" foi adicionada como opção de resposta para itens que não condizem com a realidade laboral do respondente. Por exemplo, alguns trabalhadores autônomos não possuem reuniões ou precisam lidar com colegas diariamente. Essas respostas serão computadas como missing value.

Prosseguindo para análises mais robustas, o instrumento foi novamente disponibilizado para a amostra-alvo. A Figura 1 apresenta brevemente o processo de construção do QRDT.

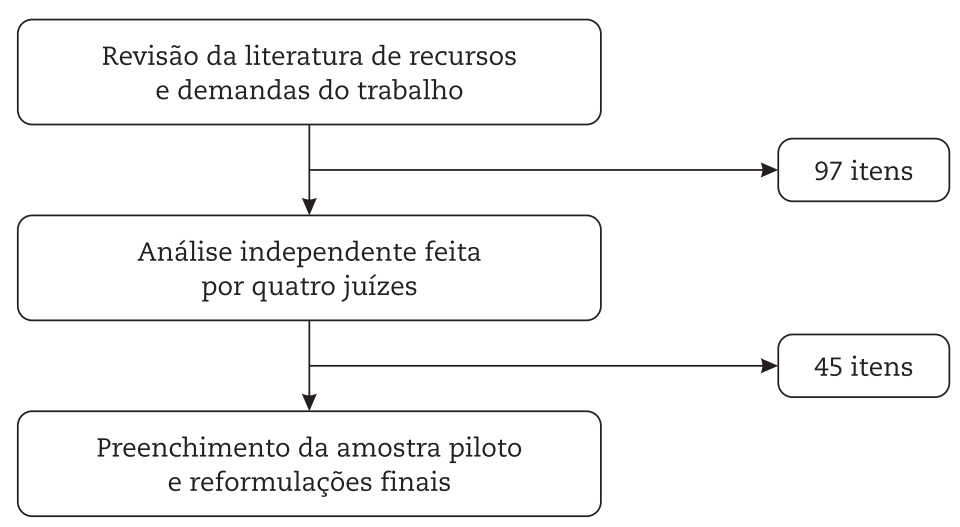

Figura 1. Processo de construção do QRDT 


\section{Procedimentos}

A coleta de dados foi realizada por meio eletrônico, utilizando-se o software de serviços de desenvolvimento de pesquisa on-line SurveyMonkey. A escala foi divulgada e disponibilizada para resposta em diferentes meios, tais como: redes sociais, e-mails e/ou convite pessoal em locais de trabalho. Foi confeccionado um convite formal para atrair participantes. O tempo médio de resposta dos participantes ao QRDT foi de cinco minutos. Foram consideradas apenas as respostas com o preenchimento completo do questionário a fim de evitar missing values.

\section{Instrumento}

Questionário de Recursos e Demandas de Trabalho - QRDT. O questionário para preenchimento da amostra-alvo conteve 45 itens, tipo Likert de quatro pontos, e se propôs a avaliar características do ambiente de trabalho relacionadas a recursos e demandas laborais percebidos por trabalhadores entre 18 e 65 anos. Nele, o respondente opta pela alternativa que mais se assemelha às suas condições de trabalho, de 1 (Discordo plenamente) até 4 (Concordo plenamente). O participante pode também responder "Não se aplica" caso nenhuma opção corresponda à sua realidade laboral. Por exemplo, itens do fator Suporte do Líder não foram respondidos por alguns trabalhadores autônomos. A alternativa "Não se aplica" é muito importante para justificar as dissonâncias entre os números de respondentes para cada fator.

\section{Análise de Dados}

Análises descritivas de cada item foram produzidas previamente no SPSS. Em um segundo momento, foi conduzida uma análise fatorial exploratória (AFE) robusta a partir da matriz de correlações policóricas dos itens do QRDT, com rotação oblimin. A AFE foi produzida a partir do software estatístico R, com método de extração minimumrank fator analysis (MRFA; Shapiro \& TenBerge, 2002). Essa metodologia de extração MRFA diminui a variância comum residual no processo de extração dos fatores e facilita a compreensão da variância comum explicada pelos fatores retidos (Shapiro \& Ten Berge, 2002). Para avaliar o ajuste da solução fatorial, utilizou-se os índices Standardized Root Mean Square Residual (SRMR) e
Goodnessof Fit Index (GFI), valores de referência são 0,08 e 0,95 , respectivamente.

Com a finalidade de determinar o número de fatores interpretados na análise fatorial, foi conduzida uma análise paralela. $\mathrm{O}$ método consiste em comparar os autovalores observados nos dados amostrais com dados provenientes de dois tipos de simulação. O primeiro método é uma simulação Monte Carlo de n matrizes de correlação com mesmo número de variáveis, enquanto o segundo método utiliza a permutação amostral em n novas amostras, em geral $n$ é igual a $\geq 500$. Após as simulações, os autovalores amostrais são comparados ao percentil 95 da média dos autovalores simulados com ambos os métodos (Timmerman \& Lorenzo-Seva, 2011).

Dois juízes independentes analisaram o conteúdo dos fatores. Quando um item apresentou carga fatorial semelhante em dois fatores, foi conduzida uma análise de conteúdo com base na literatura para averiguar qual a dimensão com que mais estava relacionado. Dessa forma, itens de carga semelhante em dois fatores foram atrelados a apenas um fator. A análise do conteúdo de cada fator e seus respectivos itens foi conduzida por dois juízes independentes, todos pós-graduados e/ou com experiência anterior em pesquisa de pelo menos dois anos.

Posteriormente, utilizou-se o software estatístico Statistical Package for the Social Sciences (SPSS) para as demais análises e cálculos estatísticos. Uma análise de fidedignidade foi conduzida para investigar a consistência interna dos fatores extraídos, por meio do coeficiente alfa de Cronbach. Dados descritivos desses fatores também foram examinados, como a média e desvio padrão. Finalmente, um teste $t$ independente foi conduzido a fim de averiguar diferenças de médias entre os sexos.

\section{Resultados}

A análise paralela sugeriu a retenção de oito fatores, os quais possuíam autovalores superiores ao percentil 95 da média dos autovalores simulados em 500 amostras, em ambos os métodos. Índices de ajuste dessa solução foram $\mathrm{SRMR}=0,04$ e GFI=0,99. Após a AFE, análises descritivas e uma análise de confiabilidade foram feitas. Seis dos oito fatores apresentaram coeficiente alfa maior que .0,80. A Tabela 1 apresenta a estrutura fatorial da QRDT.

Tabela 1

Estrutura Fatorial da QRDT

\begin{tabular}{|c|c|c|c|c|c|c|c|c|}
\hline Itens & F1 & F2 & F3 & F4 & F5 & F6 & F7 & F8 \\
\hline 14. O salário que recebo é compatível com o trabalho que realizo. & 0,44 & 0,25 & 0,05 & $-0,01$ & $-0,05$ & 0,15 & $-0,21$ & $-0,13$ \\
\hline 16. A transmissão das informações em minha equipe é eficiente. & 0,34 & 0,13 & 0,15 & 0,32 & 0,04 & $-0,11$ & 0,02 & 0,21 \\
\hline $\begin{array}{l}\text { 19. Quando são feitas mudanças no trabalho, eu sei bem como } \\
\text { elas vão funcionar na prática. }\end{array}$ & 0,50 & 0,08 & 0,18 & 0,32 & 0,12 & 0,37 & 0,04 & 0,02 \\
\hline $\begin{array}{l}\text { 26. Sou informado adequadamente dos desenvolvimentos na } \\
\text { minha organização. }\end{array}$ & 0,57 & 0,31 & - & 0,02 & 0,30 & $-0,20$ & 0,02 & 0,11 \\
\hline
\end{tabular}


Tabela 1 (continuação) Estrutura Fatorial da QRDT

Itens

30. Eu apoio as mudanças que estão ocorrendo na minha organização.

38. Eu me sinto participando das mudanças da empresa.

2. Eu recebo feedback (de colegas e/ou clientes) sobre como fazer meu trabalho.

7. Eu confio no meu líder.

13. Eu tenho um bom relacionamento com meu líder.

20. Eu recebo periodicamente feedback (elogios e/ou críticas) do meu chefe.

21. Meus líderes mostram reconhecimento pelo meu bom trabalho e pelo meu esforço extra.

31. Eu posso contar com o meu chefe/superior para me ajudar com problemas no trabalho.

34. Meu líder se interessa e incentiva minhas opiniões e ideias.

36. Meus líderes sabem coordenar pessoas e distribuir tarefas adequadamente.

6. Em minha opinião, as regras e procedimentos no trabalho são aplicados de modo correto e justo.

11. O volume de atividades permite que eu termine minhas tarefas no horário normal de trabalho.

40. No meu trabalho, diferentes grupos me exigem coisas que são difíceis de combinar.*

42. Eu tenho prazos impossíveis de serem cumpridos.*

12. Existe cooperação entre os colegas da minha equipe.

27. As relações no meu trabalho são tensas.*

33. Há conflitos pessoais entre os membros da minha equipe de trabalho.*

35. Eu posso contar com meus colegas de trabalho para me ajudar ou dar apoio quando preciso.

41. Eu me relaciono bem com meus colegas de trabalho.

44. As minhas ideias são aproveitadas pelo grupo de trabalho.

1. Eu entendo como meu trabalho se encaixa no objetivo geral da organização.

5. Está suficientemente claro para você o quê precisa fazer no seu trabalho.

8. Meu trabalho contribui para os resultados e objetivos da minha organização.

22. Eu sei bem o que é esperado de mim no trabalho.

23. Eu conheço as metas e objetivos da minha empresa.

17. Eu posso dar minha opinião sobre a forma como eu trabalho.

18. Eu disponho das informações de que necessito para fazer um bom serviço.

24. Meu trabalho combina bem com meus valores pessoais.

$\begin{array}{llllllll}\text { F1 } & \text { F2 } & \text { F3 } & \text { F4 } & \text { F5 } & \text { F6 } & \text { F7 } & \text { F8 }\end{array}$

$\begin{array}{llllllll}0,35 & 0,18 & 0,18 & 0,11 & 0,18 & -0,10 & 0,21 & 0,25\end{array}$

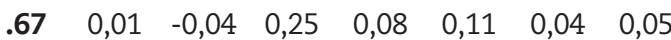

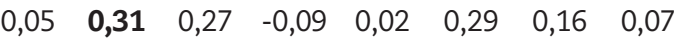

$\begin{array}{llllllll}-0,04 & \mathbf{0 , 7 8} & 0,20 & 0,07 & 0,06 & -0,20 & 0,15 & 0,09\end{array}$

$\begin{array}{llllllll}-0,18 & \mathbf{0 , 8 1} & 0,09 & 0,03 & 0,18 & -0,07 & 0,15 & 0,06\end{array}$

$\begin{array}{llllllll}0,06 & \mathbf{0 , 8 6} & -0,08 & -0,06 & -0,09 & 0,10 & -0,07 & 0,08\end{array}$

$\begin{array}{llllllll}0,08 & \mathbf{0 , 8 8} & 0,05 & -0,07 & 0,02 & 0,02 & 0,03 & 0,03\end{array}$

$\begin{array}{llllllll}-0,03 & \mathbf{0 , 8 8} & -0,09 & 0,13 & 0,04 & 0,04 & -0,04 & -0,06\end{array}$

$\begin{array}{llllllll}0,08 & \mathbf{0 , 9 2} & -0,03 & 0,07 & -0,12 & -0,01 & -0,02 & -0,03\end{array}$

$\begin{array}{lllllll}0,03 & \mathbf{0 , 8 0} & -0,04 & 0,06 & -0,02 & -0,16 & 0,13\end{array}$

$\begin{array}{llllllll}0,18 & 0,05 & \mathbf{0 , 5 2} & 0,12 & -0,14 & -0,02 & 0,20 & 0,23\end{array}$

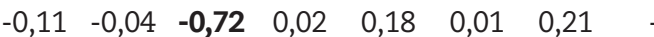

$\begin{array}{llllllll}0,33 & 0,06 & -0,48 & -0,04 & 0,31 & -0,09 & 0,09 & 0,21\end{array}$

$0,20 \quad-\quad-0,48 \quad-0,02 \quad-0,11 \quad-0,26 \quad 0,31 \quad 0,18$

$\begin{array}{llllllll}- & 0,12 & 0,23 & \mathbf{0 , 7 1} & -0,13 & -0,04 & 0,08 & 0,20\end{array}$

$\begin{array}{llllllll}- & 0,04 & 0,11 & \mathbf{0 , 6 7} & -0,16 & 0,04 & -0,09 & 0,01\end{array}$

$\begin{array}{llllllll}0,02 & 0,03 & -0,07 & \mathbf{0 , 6 2} & 0,23 & -0,11 & -0,12 & 0,12\end{array}$

$\begin{array}{llllllll}0,08 & 0,16 & -0,05 & \mathbf{0 , 7 1} & -0,16 & 0,02 & 0,06 & 0,04\end{array}$

$\begin{array}{llllllll}0,02 & 0,24 & -0,23 & \mathbf{0 , 6 4} & 0,12 & 0,27 & -0,05 & -0,15\end{array}$

$\begin{array}{llllllll}0,42 & 0,14 & 0,01 & \mathbf{0 , 4 4} & 0,03 & 0,02 & 0,05 & 0,03\end{array}$

$\begin{array}{llllllll}0,10 & 0,03 & -0,07 & 0,10 & \mathbf{0 , 7 7} & -0,04 & 0,14 & 0,03\end{array}$

$\begin{array}{llllllll}-0,16 & 0,09 & 0,24 & -0,11 & \mathbf{0 , 4 8} & 0,40 & 0,17 & -0,02\end{array}$

$\begin{array}{llllllll}-0,06 & 0,11 & -0,05 & 0,06 & \mathbf{0 , 5 6} & 0,02 & 0,45 & 0,08\end{array}$

$\begin{array}{llllllll}-0,01 & 0,13 & 0,13 & -0,09 & \mathbf{0 , 5 5} & 0,37 & -0,02 & 0,12\end{array}$

$\begin{array}{llllllll}0,37 & -0,06 & - & 0,07 & \mathbf{0 , 6 2} & 0,14 & -0,20 & 0,18\end{array}$

$\begin{array}{llllllll}0,13 & 0,21 & 0,24 & 0,13 & 0,03 & \mathbf{0 , 4 1} & 0,05 & -0,03\end{array}$

$\begin{array}{llllllll}0,10 & 0,06 & 0,25 & -0,01 & 0,15 & \mathbf{0 , 5 2} & -0,06 & 0,09\end{array}$

$\begin{array}{llllllll}0,18 & -0,06 & 0,07 & 0,02 & 0,20 & \mathbf{0 , 6 1} & 0,12 & -0,04\end{array}$ 
Tabela 1 (continuação) Estrutura Fatorial da QRDT

\begin{tabular}{|c|c|c|c|c|c|c|c|c|}
\hline Itens & F1 & F2 & F3 & F4 & F5 & F6 & F7 & F8 \\
\hline $\begin{array}{l}\text { 25. Meu trabalho proporciona desafios e possibilidades de } \\
\text { aprendizado. }\end{array}$ & $-0,02$ & 0,01 & $-0,14$ & 0,09 & 0,04 & 0,68 & 0,26 & 0,16 \\
\hline 28. Eu posso escolher como fazer o meu trabalho. & 0,06 & $-0,05$ & 0,09 & $-0,02$ & $-0,08$ & 0,69 & 0,15 & $-0,05$ \\
\hline 29. Meu trabalho atual combina bem com o que posso realizar. & $-0,06$ & $-0,09$ & $-0,03$ & 0,14 & 0,11 & 0,77 & $-0,01$ & 0,12 \\
\hline $\begin{array}{l}\text { 39. Eu tenho oportunidades suficientes no trabalho para usar } \\
\text { minhas habilidades e capacidades. }\end{array}$ & 0,16 & 0,01 & 0,01 & 0,18 & $-0,07$ & 0,67 & 0,08 & 0,11 \\
\hline $\begin{array}{l}\text { 43. Meus valores pessoais estão alinhados com os da organização } \\
\text { para a qual eu trabalho. }\end{array}$ & 0,35 & $-0,10$ & 0,10 & 0,24 & 0,24 & 0,35 & 0,03 & 0,10 \\
\hline $\begin{array}{l}\text { 45. Eu recebo solicitações de tarefas sempre de acordo com minha } \\
\text { função. }\end{array}$ & 0,04 & 0,24 & - & 0,16 & $-0,15$ & 0,37 & $-0,23$ & 0,12 \\
\hline 9. O trabalho que eu realizo me exige muito. & $-0,03$ & 0,02 & $-0,16$ & $-0,07$ & 0,04 & 0,18 & 0,78 & $-0,05$ \\
\hline $\begin{array}{l}\text { 10. Eu participo da tomada de decisão sobre assuntos } \\
\text { relacionados diretamente com meu trabalho. }\end{array}$ & 0,43 & $-0,08$ & 0,25 & 0,02 & $-0,10$ & 0,23 & 0,43 & 0,11 \\
\hline 15. Eu desempenho diferentes tipos de tarefas no meu trabalho. & 0,09 & 0,03 & $-0,06$ & 0,04 & 0,12 & 0,12 & 0,55 & $-0,01$ \\
\hline 3. Meu trabalho tem oportunidades de promoção. & $-0,05$ & 0,02 & 0,03 & 0,04 & 0,02 & $-0,03$ & $-0,02$ & 0,92 \\
\hline $\begin{array}{l}\text { 4. A empresa oferece oportunidades para meu crescimento } \\
\text { profissional. }\end{array}$ & $-0,02$ & 0,05 & 0,04 & 0,13 & - & 0,06 & 0,07 & 0,82 \\
\hline $\begin{array}{l}\text { 37. Eu tenho um plano de carreira dentro da organização para a } \\
\text { qual trabalho. }\end{array}$ & 0,02 & 0,19 & $-0,17$ & $-0,26$ & 0,01 & 0,15 & $-0,15$ & 0,72 \\
\hline Eigenvalues & 3,27 & 6,45 & 2,20 & 3,74 & 3,27 & 4,97 & 2,25 & 3,23 \\
\hline \% Variância Comum Explicada & 11 & 22 & 7 & 13 & 11 & 17 & 8 & 11 \\
\hline$\alpha$ & 0,81 & 0,92 & 0,57 & 0,81 & 0,81 & 0,89 & 0,45 & 0,80 \\
\hline Média & 2,91 & 2,98 & 2,76 & 3,10 & 3,60 & 3,33 & 3,45 & 2,73 \\
\hline Desvio Padrão & 0,72 & 0,81 & 0,69 & 0,66 & 0,52 & 0,62 & 0,54 & 0,95 \\
\hline
\end{tabular}

Notas. o asterisco $\left({ }^{*}\right)$ sinaliza itens invertidos; F=Fator

Por fim, um teste $t$ foi realizado para avaliar diferenças significativas entre os sexos nos fatores da QRDT. Não houve diferenças significativas de médias nos fatores do QRDT entre os sexos. A magnitude observada do $d$ de
Cohen ficou abaixo de 0,18 nos oito fatores avaliados, o que indica diferenças pequenas na percepção de recursos e demandas de trabalho entre homens e de mulheres. A Tabela 2 apresenta o resultado dos testes $t$.

Tabela 2

Diferenças entre Sexos dos Fatores do QRDT

\begin{tabular}{|c|c|c|c|c|c|}
\hline \multirow{2}{*}{ Fatores } & Homens & Mulheres & \multirow{2}{*}{$d$} & \multirow{2}{*}{$t$} & \multirow[b]{2}{*}{$p$} \\
\hline & $M(D P)$ & $M(D P)$ & & & \\
\hline 1. Comunicação & $2,99(0,64)$ & $2,87(0,76)$ & 0,17 & $-1,47$ & 0,14 \\
\hline 2. Suporte do Líder & $2,91(0,73)$ & $3,01(0,85)$ & $-0,13$ & 1 & 0,32 \\
\hline 3. Justiça no Trabalho & $2,72(0,70)$ & $2,78(0,68)$ & $-0,09$ & 0,72 & 0,47 \\
\hline 4. Suporte dos Colegas & $3,12(0,58)$ & $3,09(0,70)$ & 0,05 & $-0,44$ & 0,66 \\
\hline 5. Clareza de Papel & $3,60(0,55)$ & $3,60(0,51)$ & 0 & 0,14 & 0,89 \\
\hline 6. Alinhamento de Habilidades & $3,32(0,58)$ & $3,33(0,64)$ & $-0,02$ & 0,05 & 0,96 \\
\hline 7. Desafios & $3,49(0,55)$ & $3,43(0,53)$ & 0,11 & $-0,90$ & 0,37 \\
\hline 8. Perspectiva de Crescimento & $2,62(0,90)$ & $2,79(0,96)$ & $-0,18$ & 1,43 & 0,15 \\
\hline
\end{tabular}




\section{Discussão}

A Figura 2 apresenta sucintamente as mudanças ocorridas no questionário baseadas nos estudos estatísticos. Uma alteração importante foi feita: a partir das análises descritivas, o item "Eu tenho pouco trabalho para fazer." foi excluído. Isso porque das 292 pessoas que responderam a esse item, 207 (70,9\%) marcaram "Discordo totalmente", e outras 46 pessoas $(15,8 \%)$ "Discordo em parte". Assim, sendo que a maioria das pessoas da amostra discordou que trabalha pouco, esse item foi excluído por dois motivos. O primeiro motivo foi por influenciar resultados anômalos, que haviam sido observados na análise inicial. O segundo foi por não gerar nenhuma informação relevante - já que parece comum a percepção do trabalho como um processo mais longo e custoso.

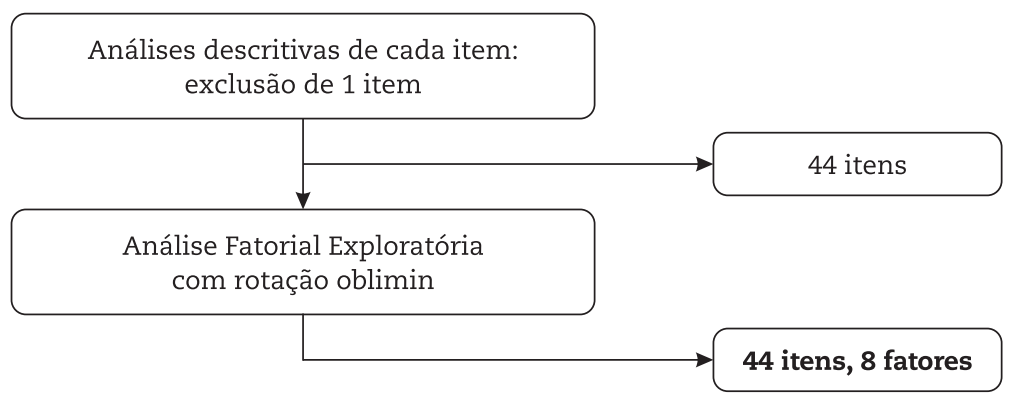

Figura 2. Mudanças na QRDT a partir das análises estatísticas

Esse resultado já era esperado devido ao baixo número de itens do QRDT. Abaixo, os fatores extraídos da AFE, seu conceito e exemplo de itens. Fator 1, Comunicação: trata das informações proporcionadas para se alcançar objetivos e estimular o desenvolvimento (Cousins et al., 2004; Mäkikangas et al., 2010; Schaufeli et al., 2009). Esse fator relaciona-se ao que é comunicado na empresa tanto em momentos de transformação organizacional quanto em momentos mais típicos. "A transmissão das informações em minha equipe é eficiente." e "Eu me sinto participando das mudanças da empresa." são, por exemplo, dois itens desse fator.

Fator 2, Suporte do Líder: o quanto o líder inspira, encoraja e conecta sua equipe, estimulando entusiasmo, responsabilidade e colaboração (Bakker, 2005; Bakker \& Demerouti, 2014; Csikszentmihalyi, 1999; Nielsen \& Cleal, 2010; Schaufeli \& Bakker, 2004). Como exemplo disso, os seguintes itens: "Eu confio no meu líder." e "Eu recebo periodicamente feedback (elogios e/ou críticas) do meu chefe."

Fator 3, Justiça no Trabalho: refere-se ao tratamento igualitário por parte dos colegas, da empresa e dos líderes nas relações de trabalho. Isso inclui uma carga de trabalho adequada além de exigências e procedimentos bem alinhados (Oliveira \& Ferreira, 2016; Schaufeli et al., 2013). O item "Em minha opinião, as regras e procedimentos no trabalho são aplicados de modo correto e justo." resume bem a ideia desse fator.

Fator 4, Suporte dos Colegas: o quanto os profissionais entendem que seus pares os apoiam e estão disponíveis para ajudá-los ou colaborar em serviços complexos (Cousins et al., 2004; Mäkikangas et al., 2010; Schaufeli
\& Bakker, 2004). A pontuação baixa no construto indicaria relações não cooperativas ou até abusivas. "Existe cooperação entre os colegas da minha equipe.” é o exemplo de um item do polo positivo desse fator. No polo negativo, o item "Há conflitos pessoais entre os membros da minha equipe de trabalho" - que tem seu escore invertido para análise dos dados.

Fator 5, Clareza de Papel: refere-se ao entendimento com nitidez pelo funcionário de suas atribuições e condutas requeridas dentro da empresa aliado ao sentimento de que as atividades realizadas geram impacto na sua vida ou no trabalho de seus colegas e de outras pessoas (Bakker \& Demerouti, 2014; Cousins et al., 2004; Maciel \& Camargo, 2016; Nielsen \& Cleal, 2010; Schaufeli et al., 2013). O funcionário compreende bem o que é esperado dele, e compreende como seu trabalho afeta a empresa - como bem resume os itens "Eu sei bem o que é esperado de mim no trabalho." e "Eu conheço as metas e objetivos da minha empresa.".

Fator 6, Alinhamento de Habilidades: trata da compreensão de que a função no trabalho combina com as capacidades e habilidades pessoais do colaborador (Bakker \& Demerouti, 2014; Mäkikangas et al., 2010; Nielsen \& Cleal, 2010). Esse fator diz respeito ao quão bem alinhado o cargo está para um funcionário - desde valores pessoais congruentes com a empresa até a autonomia para explorar e executar tarefas. Por tal razão, o item 43 ("Meus valores pessoais estão alinhados com os da organização para a qual eu trabalho.") - que possui carga fatorial semelhante em dois fatores - permanece nesse fator, e não em Comunicação. Um exemplo de uma afirmação dessa dimensão é o item "Eu tenho 
oportunidades suficientes no trabalho para usar minhas habilidades e capacidades.".

Fator 7, Desafios: equilíbrio entre as capacidades da pessoa e as determinações organizacionais, fazendo do dia a dia desafiador na medida certa, ou seja, nem muito acima e nem muito abaixo das potencialidades do indivíduo (Csikszentmihalyi, 1999; Nielsen \& Cleal, 2010; Schaufeli et al., 2013). O item 10 - "Eu participo da tomada de decisão sobre assuntos relacionados diretamente com meu trabalho." - possui carga fatorial semelhante em Comunicação. Já que o conteúdo desse item está mais relacionado à capacidade de realizar atribuições laborais e, assim, mais próximo da definição da dimensão "Desafios", decidiu-se por incluí-lo nesse fator. "Eu desempenho diferentes tipos de tarefas no meu trabalho." é outro item que compõe essa dimensão.

Fator 8, Perspectiva de Crescimento: diz respeito ao entendimento de que o trabalho pode ser constituído a fim de possibilitar o desenvolvimento, o crescimento e a aprendizagem dos indivíduos (Csikszentmihalyi, 1999; Mäkikangas et al., 2010). As frases construídas em cada item dessa dimensão descrevem oportunidades disponíveis na empresa e planejamento para alcançá-las, por exemplo: "Eu tenho um plano de carreira dentro da organização para a qual trabalho".

É interessante notar que todos os fatores tratam de recursos de trabalho. Assim, quanto mais uma pessoa pontua numa dimensão, mais esse fator parece estar presente em seu ambiente laboral. Portanto, uma pontuação baixa em quaisquer fatores do QRDT pode indicar a presença de uma demanda de trabalho.

Este estudo teve como propósito construir e validar o Questionário de Recursos e Demandas de Trabalho. $\mathrm{O}$ instrumento foi baseado no modelo de Recursos e Demandas de Trabalho (Bakker \& Demerouti, 2014; Schaufeli \& Bakker, 2004; Schaufeli et al., 2013) e demonstrou aspectos psicométricos adequados. O questionário final possui 44 itens divididos em oito fatores. Dois fatores, entretanto, apresentaram coeficientes alfa considerados baixos. Esses fatores são Justiça no Trabalho $(n=253 ; \alpha=0,57)$, com 4 itens; e Desafios $(n=280$; $\alpha=0,45$ ), com três itens. Ressalta-se aqui que um coeficiente dessa magnitude não necessariamente indica má precisão na mensuração do construto (Field, 2013), sendo que o baixo número de itens pode ter influenciado tal escore (Cronbach, 1957).

Em concordância com Gleitman, Reiberg e Gross (2009), seria fundamental repetir a aplicação desse questionário a um maior número amostral, em diferentes estados do Brasil ou até em diferentes países. Isso permite uma melhor compreensão da distribuição de respostas aos itens e possibilita uma validade mais robusta do questionário construído. Com isso em mente, espera-se que o QRDT possa ser útil na mensuração de recursos e demandas de trabalho em ambientes laborais de todo país.

\section{Referências}

Bakker, A. B. (2005). Flow among music teachers and their students: The crossover of peak experiences. Journal of Vocational Behavior, 66(1), 26-44. doi: 10.1016/j.jvb.2003.11.001

Bakker, A. B., \& Demerouti, E. (2014). Job Demands-Resources Theory. Wellbeing, III, 1-28. doi: 10.1002/9781118539415.wbwell019

Bakker, A. B., Van Veldhoven, M., \& Xanthopoulou, D. (2010). Beyond the Demand-Control Model: Thriving on high job demands and resources. Journal of Personnel Psychology, 9(1), 3-16. doi: 10.1027/1866-5888/a000006

Cervo, C. S., Mónico, L. S. M., Santos, N. R., Hutz, C. S., \& Pais, L. (2016). Authentic Leadership Questionnaire: Invariance between samples of Brazilian and Portuguese employees. Psicologia: Reflexão e Crítica, 29(1), 40-51. doi: 10.1186/s41155-016-0046-4

Cousins, R., Mackay, C. J., Clarke, S. D., Kelly, C., Kelly, P. J., \& McCaig, R. H. (2004).'Management standards' work-related stress in the UK: Practical development. Work \& Stress, 18(2), 113-136. doi: 10.1080/02678370410001734322

Cronbach, L. J. (1951).Coefficient alpha and the internal structure of tests. Psychometrika, 16(3), 297-334. doi: 10.1007/BF02310555

Csikszentmihalyi, M., (1999). If we are so rich, why aren't we happy? American Psychologist Associtation, 54(10), 821-827.

Dal Vesco, D. G., Beuren, I. M., \& Popik, F. (2016). Percepção de justiça na avaliação na avaliação de desempenho e satisfação do trabalho. Enfoque: Reflexão Contábil, 35(3), 121-138. doi: 10.4025/enfoque.v35i3.28333

Erdogan, B., Bauer, T. N., Truxillo, D. M., \& Mansfield, L. R. (2012).Whistle while you work: A review of the life satisfaction literature. Journal of Management, 38(4), 1038-1083. doi: 10.1177/0149206311429379

Farina, L. S. A., Rodrigues, G. R., \& Hutz, C. S. (2018). Flow and Engagement at Work: A Literature Review. Psico-USF, $23(4), 633-642$. doi: 10.1590/1413-82712018230404

Field, A. (2013). Discovering statistics using IBM SPSS Statistics: And sex and drugs and rock ' $n$ ' roll (4th edition). London: Sage.

Filho, A. L. A. S., Ferreira, M. C., \& Valentini, F. (2017). Evidências de validade da Escala de Consciência Plena no Trabalho (ECPT). Psico, 48(3), 2017. doi: 10.15448/1980-8623.2017.3.24034

Gleitman, H., Reiberg, D., \& Gross, J. (2009). Psicologia. Porto Alegre: Artmed.

Hakanen, J. J., \& Schaufeli, W. B. (2012). Do burnout and work engagement predict depressive symptoms and life satisfaction? A three-wave seven-year prospective study. Journal of Affective Disorders, 141(1), 415-424. doi: 10.1016/j.jad.2012.02.043

International Test Commission (2005). International Guidelines on Test Adaptation. [www.intestcom.org]

Jesus, R. G., \& Rowe, D. E. O. (2014). Justiça organizacional percebida por professores do ensino básico, técnico e tecnológico. Revista de Administração Mackenzie, 15(6), 172-200. doi: 10.1590/1678-69712014/administracao.v15n6p172-200 
Maciel, C. O., \& Camargo, C. (2016). Social connection in organizations: The effects of local ties on job engagement and performance. Revista de Administração (São Paulo), 51(4), 377-385. doi: 10.1016/j.rausp.2016.07.005

Mäkikangas, A., Bakker, A. B., Aunola, K., \& Demerouti, E. (2010). Job resources and flow at work: Modelling the relationship via latent growth curve and mixture model methodology. Journal of Occupational and Organizational Psychology, 83(3), 795-814. doi: 10.1348/096317909X476333

Nielsen, K., \& Cleal, B. (2010). Predicting flow at work: Investigating the activities and job characteristics that predict flow states at work. Journal of Occupational Health Psychology, 15(2), 180-190. doi: 10.1037/a0018893

Nota, L., \& Rossier, J. (2015). Handbook of Life Design: From Practice to Theory and from Theory to Practice. Boston: Hogrefe.

Oliveira, D. D. F., \& Ferreira, M. C. (2016). O impacto das percepções de justiça organizacional e da resiliência sobre o engajamento no trabalho [The impact of organizational justice perceptions and resilience on the work engagement]. Estudos de Psicologia, 33(4), 747755. doi: 10.1590/1982-02752016000400017

Reijseger, G., Schaufeli, W. B., Peeters, M. C. W., Taris, T. W., Beek, I. van, \& Ouweneel, E. (2013). Watching the paint dry at work: Psychometric examination of the Dutch Boredom Scale. Anxiety, Stress \& Coping: An International Journal, 26(5), 508-525, doi: $10.1080 / 10615806.2012 .720676$

Shapiro, A., \& Ten Berge, J. M. F. (2002). Statistical inference of minimum rank factor analysis. Psychometrika, 67(1), 79-94.

Schaufeli, W., \& Bakker, A. B. (2004). Job Demands, Job Resources and Their Relationship with Burnout and Engagement : A MultipleSample Study. Journal of Organizational Behavior, 25, 293-315. doi: 10.1002/job.248

Schaufeli, W. B., Bakker, A. B., Hoogduin, K., Schaap, C., \& Kladler, A. (2001). On the clinical validity of the Maslach Burnout Inventory and the Burnout Measure. Psychology and Health, 16(5), 565-582. doi: 10.1080/08870440108405527

Schaufeli, W., Bakker, A. B., Van Rhenen, W. (2009). How changes in job demands and resources predict burnout, work engagement, and sickness absenteeism. Journal of Organizational Behavior, 30(7), 893-917. doi: 10.1002/job.595

Schaufeli, W., Dijikstra, P., \& Vazquez, A. C. (2013). Engajamento no Trabalho. São Paulo: Casa do Psicólogo.

Schaufeli, W. B. (2017). Applying the Job Demands-Resources model: A 'how to' guide to increase work engagement and prevent burnout. Organizational Dynamics, 2(46), 120-132. doi: 10.1016/j.orgdyn.2017.04.008

Slemp, G. R., \& Vella-Brodrick, D. A., (2013). The job crafting questionnaire: A new scale to measure the extent to which employees engage in job crafting. International Journal of Wellbeing, 3(2), 126-146. doi:10.5502/ijw.v3i2.1

Snyder, C. R., \& Lopez, S. J. (2009). Psicologia Positiva. Porto Alegre: Artmed.

Taris, T. W., \& Schaufeli, W. B. (2016).The Job Demands-Resources model. Em S. Clarke, T. M. Probst, F. Guldenmund \& J. Passmore (Eds.). The Wiley Blackwell handbook of the psychology of occupational safety and workplace health (157-180). Chichester: John Wiley.

Timmerman, M. E., \& Lorenzo-Seva, U. (2011). Dimensionality assessment of ordered polytomousitens with parallel analysis. Psychological Methods, 16(2), 209-220. doi: 10.1037/a0023353

VanVeldhoven, M., \& Meijman, T., (1994). Het meten van psychosocial airbeds belasting met een vragenlijst: de vragenlijst beleving en beoordeling van de arbeid (VBBA) [Measuring psychosocial workload with a survey: The questionnaire on the experience and evaluation of work (QEEW)]. Amsterdam: NIA.

Vazquez, A. C. S., Magnan, E. dos S., Pacico, J. C., Hutz, C. S., \& Schaufeli, W. B. (2016). Avaliação do engajamento das pessoas com o seu trabalho: A versão brasileira da escala Utrecht de engajamento no trabalho (UWES). Em Hutz, C. S. (Eds.). Avaliação em Psicologia Positiva (75-89). São Paulo: Hogrefe.

\section{Sobre os autores}

Larissa Sanford Ayres Farina é Psicóloga, formada pela Universidade Federal do Rio Grande do Sul (UFRGS), Especialista em Gestão de Pessoas e em Dinâmica dos Grupos, Mestra e atual Doutoranda do Programa de Pós-Graduação em Psicologia também pela UFRGS.

Gabriel dos Reis Rodrigues é Graduando em Psicologia pela Universidade Federal do Rio Grande do Sul (UFRGS). Atualmente, é Bolsista de Iniciação Científica no Laboratório de Mensuração (Instituto de Psicologia - UFRGS).

Natália Kluwe Fagundes é Graduanda em Psicologia pela Universidade Federal do Rio Grande do Sul (UFRGS), tendo atuado como Bolsista de Iniciação Científica no Laboratório de Mensuração (Instituto de Psicologia - UFRGS).

Thamires Casarotto Carafini é Graduanda na Universidade Luterana do Brasil no curso de Psicologia. É Bolsista Voluntária de Iniciação Científica no Laboratório de Mensuração (Instituto de Psicologia - UFRGS).

Laisla Gabriele Conceição Soares Moreira é Graduanda em Psicologia pela Faculdade de Desenvolvimento do Rio Grande do Sul. É Bolsista Voluntária de Iniciação Científica no Laboratório de Mensuração (Instituto de Psicologia - UFRGS).

Wagner De Lara Machado é Psicólogo com Pós-Doutorado como consultor de análise quantitativa de dados e psicometria (UFRGS). É Professor Titular na PUCRS e coordena o Grupo de Pesquisa de Avaliação em Bem-estar e Saúde Mental.

Claudio Simon Hutz é Psicólogo com Doutorado na University of Iowa (USA) e Pós-Doutorado na Arizona State University (USA). É Professor Titular da UFRGS e coordenador do Laboratório de Mensuração (Instituto de Psicologia - UFRGS). 